\title{
Self Adaptive Contention Aware Routing Protocol for Intermittently Connected Mobile Networks
}

\author{
Ahmed Elwhishi, Pin-Han Ho, K. Naik, and Basem Shihada
}

\begin{abstract}
This paper introduces a novel multi-copy routing protocol, called Self Adaptive Utility-based Routing Protocol (SAURP), for Delay Tolerant Networks (DTNs) that are possibly composed of a vast number of devices in miniature such as smart phones of heterogeneous capacities in terms of energy resources and buffer spaces. SAURP is characterized by the ability of identifying potential opportunities for forwarding messages to their destinations via a novel utility function based mechanism, in which a suite of environment parameters, such as wireless channel condition, nodal buffer occupancy, and encounter statistics, are jointly considered. Thus, SAURP can reroute messages around nodes experiencing high buffer occupancy, wireless interference, and/or congestion, while taking a considerably small number of transmissions. The developed utility function in SAURP is proved to be able to achieve optimal performance, which is further analyzed via a stochastic modeling approach. Extensive simulations are conducted to verify the developed analytical model and compare the proposed SAURP with a number of recently reported encounter-based routing approaches in terms of delivery ratio, delivery delay, and the number of transmissions required for each message delivery. The simulation results show that SAURP outperforms all the counterpart multi-copy encounter-based routing protocols considered in the study.
\end{abstract}

Index Terms-Encounter-based Routing, DTN.

\section{INTRODUCTION}

Delay Tolerant Network (DTN) [1] is characterized by the lack of end-to-end paths for a given node pair for extended periods, which poses a completely different design scenario from that for conventional mobile adhoc networks (MANETs) [13]. Due to the intermittent connections in DTNs, a node is allowed to buffer a message and wait until the next hop node is found to continue storing and carrying the message. Such a process is repeated until the message reaches its destination. This model of routing is significantly different from that employed in the MANETs. DTN routing is usually referred to as encounter-based, store-carry-forward, or mobility-assisted routing, due to the fact that nodal mobility serves as a significant factor for the forwarding decision of each message.

Depending on the number of copies of a message that may coexist in the network, two major categories of encounter-based routing schemes are defined: singlecopy and multi-copy. With the single-copy schemes [5], no more than a single copy of a message can be carried by any node at any instance. Although simple and resource efficient, the main challenge in the implemen-

- Ahmed Elwhishi, Pin Han Ho, and K. Naik are with the Department of Electrical and Computer Engineering, University of Waterloo, Ontario, Canada.

E-mail: snaik@uwaterloo.ca.

- Basem Shihada. is with Department of Computer Science, KAUST, Thuwal, Saudi Arabia.

E-mail: basem.shihada@kaust.edu.sa tation of single-copy schemes lies in how to effectively deal with the interruptions of network connectivity and node failures. Thus, single-copy schemes have been reported to seriously suffer from long delivery delays and/or large message loss ratio. On the other hand, multiple-copy (or multi-copy) routing schemes allow the networks to have multiple copies of the same message that can be routed independently and in parallel so as to increase robustness and performance. It is worth noting that most multi-copy routing protocols are floodingbased [3], [4] that distribute unlimited numbers of copies throughout the network, or controlled flooding-based [20], [7] that distribute just a subset of message copies, or utility-based approaches [2] that determine whether a message should be copied to a contacted node simply based on a developed utility function.

Although improved in terms of performance, the previously reported multi-copy schemes are subject to the following problems and implementation difficulties. First, these schemes inevitably take a large number of transmissions, energy consumption, and a vast amount of transmission bandwidth and nodal memory space, which could easily exhaust the network resource. Second, they suffer from contention in case of high traffic loads, when packet drops could result in a significant degradation of performance and scalability. Note that the future DTNs are expected to operate in an environment with a large number of miniature hand-held devices such as smart phones, tablet computers, personal digital assistants (PDAs), and mobile sensors. In such a scenario, it may no longer be the case that nodal contact frequency serves as the only dominant factor for the 
message delivery performance as that assumed by most existing DTN literature. Therefore, limitations on power consumption, buffer spaces, and user preferences should be jointly considered in the message forwarding process.

To cope with the above-mentioned deficiency, a family of multi-copy schemes called utility-based controlled flooding [15], [21], [14], [12] has been proposed. The class of schemes generate only a small number of copies to ensure that the network is not overloaded with the launched messages. Although being able to effectively reduce the message delivery delay and the number of transmissions, most of the utility-based controlled flooding routing schemes in literature assume that each node has sufficient resources for message buffering and forwarding. None of them, to our best knowledge, has sufficiently investigated how the protocol should take advantage of dynamic network status to improve the performance, such as packet collision statistics, wireless link conditions, nodal buffer occupancy, and battery status. Note that the nodal buffer status could serve as an indicator how much the opportunity cost is by accepting a forwarded message, while the channel condition is an indicator how likely the contact could be an eligible one; or in other words, how likely a message can be successfully forwarded during the contact. They are obviously essential parameters to be considered in the utility function.

With this in mind, we introduce a novel DTN routing protocol, called Self Adaptive Utility-based Routing Protocol (SAURP), that aims to overcome the shortcomings of the previously reported multi-copy schemes. Our goal is to achieve a superb applicability to the DTN scenario with densely distributed hand-held devices. The main feature of SAURP is the strong capability in adaptation to the fluctuation of network status, traffic patterns/characteristics, user encounter behaviors, and user resource availability, so as to improve network performance in terms of message delivery ratio, message delivery delay, and number of transmissions.

The contributions of the paper are as following

- We develop a novel DTN routing scheme which incorporates with some parameters that have not been jointly considered in the literature. The parameters include link quality/availability and buffer occupancy statistics, which are obtained by sampling the channels and buffer space during each contact with another node.

- We introduce a novel transitivity update rule, which can perfectly match with the proposed routing model and the required design premises.

- We introduce a novel adaptive time-window update strategy for maintaining the quality metric function at each node, aiming at an efficient and optimal decision making process for each active data message.

- An analytical model is developed for the proposed SAURP, and its correctness is further verified. We show via extensive simulations that the proposed SAURP can achieve significant performance gain over the previously reported counterparts under the considered scenarios.

The rest of the paper is organized as follows. Section II provides a review of the related work. Section III describes the proposed SAURP in detail. Then, Section IV analyzes the proposed SAURP by coming up with an analytical model for the delivery ratio and end-to-end message delivery delay. Section V provides the simulation results and comparisons with the counterparts. In Section VI, we conclude the paper.

\section{Related WORK}

Most (if not all) previously reported encounter-based routing schemes have focused on nodal mobility, which has been extensively exploited as the dominant factor in the message forwarding decision. Those schemes contributed in the context of introducing new interpretations of the observed node mobility in the per-node utility function. Spyropoulos et al. in [6], [12] developed routing strategies that use different utility routing metrics based on nodal mobility statistics, namely Most Mobile First (MMF), Most Social First (MSF), and Last Seen First (LSF). Nelson et al. [29] proposed an enhanced version of MSF by taking the number of message replicas transferred during each contact in proportion to the pernode utility function, which is in turn determined by the evolution of the number of nodal encounters during each time-window. Lindgren et al. in Jones et al. in [19] introduced a novel utility function for DTN routing by manipulating the minimum expected inter-encounter duration between nodes. Ling et al. in [23] designed a feedback adaptive routing scheme based on the influence factors solely determined by the node mobility, where a node with higher mobility is given a higher factor, and messages are transmitted through nodes with higher influence factors.

Lindgren et al. in [2] introduced a DTN routing scheme which predicts encounter probability between nodes. Burgess et al. in [38] introduced a routing protocol which bases its decisions on whether to transmit or delete a message on the path likelihood. The path likelihood metric is based on historic information of the number of encounters between nodes. Y. Liao et al. in [37] introduced a routing scheme that combines erasurecoding with an estimation routing scheme and selectively distributes messages blocks to relay nodes. The decision of forwarding a message depends on the contact frequency and other factors such as buffer occupancy, and available battery power level.

Balasubramanian et al. in [22] introduced a routing scheme as resource allocation. The statistics of available bandwidth and the number of message replicas currently in the network are considered in the derivation of the routing metric to decide which message to replicate first among all the buffered messages in the custodian node. The derivation of the routing metric, nonetheless, is not related to buffer status. Along the similar line 
of research, Khrifa et al. in [43] proposed a forwarding and dropping policy for a limited buffer capacity. The decision under this policy is made based on the value of per-message marginal utility. This policy nonetheless was designed to suit homogeneous nodal mobility. Lee et al. in [39] introduced a comprehensive routing scheme as resource allocation that jointly optimizes link scheduling, routing, and replication. This framework allows the developed solutions to be adaptive to various network conditions regarding nodal interferences and connections/disconnections. Tan et al. in [36] introduced a routing strategy based on calculating the expected endto-end path length as a metric in forwarding messages mainly based on the reciprocal of the encountering probability. It is defined as the expectation of message transmission latency through multi-hop relays.

Another scheme called delegation forwarding was introduced in [14], where a custodian node forwards a message copy to an encountered node if the encountered node has a better chance to "see" the destination. The key idea is that a custodian node (source or relay) forwards a message copy only if the utility function (represented by the rate of encounters between node pairs) of the encountered node is higher than all the nodes so far "seen" by a message, and then current custodian will update its utility value of that message to be equal to that of the encountered node. Spyropoulos et al. in [6] propsed routing scheme called Spray and Focus, which is characterized by addressing an upper bound on the number of message copies (denoted as $L$ ). In specific, a message source starts with $L$ copy tokens. When it encounters another node $B$ currently without any copy of the message, it shares the message delivery responsibility with $B$ by transferring $L / 2$ of its current tokens to $B$ while keeping the other half for itself. When it has only one copy left, it switches to a utility forwarding mechanism based on the time elapsed since the last contact. This scheme has proven to significantly reduce the required number of transmissions, while achieving a competitive delay with respect to network contentions such as buffers space and bandwidth.

Some studies have investigated the impact of human mobility and their social relations on the routing algorithms [32], [33], [34], [35], [31], [17], [41], leading to a class of social network based message forwarding schemes. With these schemes, the variation in node popularity and the detectability of communities are employed as the main factors in the forwarding decisions. Bubble-rap [34] is a representative protocol by considering the importance of individuals in a social network for making the message forwarding decision. Mosli et al. in[17] introduced a DTN routing scheme using utility functions calculated from an evaluation of context information. The derived cost function is used as an assigned weight for each node that quantifies its suitability to deliver messages to an encountered node regarding a given destination. Other schemes are based on content-based network service [40] as a novel style of communication that associates source and destination pairs based on actual content and interests, rather than by letting the source to specify the destination.

Although previously reported studies such as [6], [7], [2], [14], [31], [22] have made great efforts in improving the DTN routing techniques, they are subject to various limitations in the utility function update processes. The schemes such as [2], [38] that take the number of encounters as the main factor in the message forwarding decision, may suffer from multiple falsely detected contacts. This happens when a node exhibits an intermittent connection with another node, e.g., due to a communication barrier. Further, a permanent or quasi-permanent neighbor will cause the utility function calculation invalid in message forwarding. For example, if node $\mathrm{A}$ and $\mathrm{B}$ remain in the transmission range of each other for a long period without disconnection, there will be only one contact counted between the two nodes irrespective of the long duration of the contact. A routing decision based on the number of contacts makes node $B$ a less suitable candidate for carrying a message from node $\mathrm{A}$ than other nodes that have a larger number of contacts, even though node B could actually be the preferred candidate for carrying the messages.

Although the abovementioned schemes can capture the mobility properties in order to come up with efficient forwarding strategy, they may not be able to acquire accurate knowledge about network dynamics and unpredicted contacts. More importantly, the channel capacity and buffer occupancy status have never been jointly considered in the derivation of utility functions for hop-by-hop message forwarding. It is clear that these two factors could only be overlooked/ignored when the encounter frequency is low since the routing protocol performance is dominated by node mobility, while the network resource availability does not play an important role. However, in the scenario that the nodal encounter frequency is large and each node has many choices for message forwarding in a short time, the network resource availability is envisioned to serve as a critical factor for performance improvement and should be utilized in the derivation of utility functions.

Motivated by the above observations, this paper investigates encounter-based routing that jointly considers nodal contact statistics and network status including wireless channel condition and buffer occupancy. Our goal is to reduce the delivery delay and the number of transmissions under stringent buffer space and link capacity constraints. This is a desired feature of a DTN especially in the scenario where each mobile node is hand-held device with limited resources.

\section{Self Adaptive Utility-based Routing PROTOCOL (SAURP)}

The proposed SAURP is characterized by the ability of adapting itself to the observed network behaviors, 
which is made possible by employing an efficient timewindow based update mechanism for some network status parameters at each node. We use time-window based update strategy because it is simple in implementation and robust against parameter fluctuation. Note that the network conditions could change very fast and make a completely event-driven model unstable. Fig. 1 illustrates the functional modules of the SAURP architecture along with their relations.

The Contact Statistics (denoted as $C S^{(i)}$ ) refers to the statistics of total nodal contact durations, channel condition, and buffer occupancy state. These values are collected at the end of each time window and used as one of the two inputs to the Utility-function Calculation and Update Module (UCUM). Another input to the UCUM, as shown in Fig. 1, is the updated utility denoted by $\triangle T_{\text {new }}^{(i)}$, which is obtained by feeding $\triangle T^{(i)}$ ( the inter-contact time between any node pair, $A$ and $B$ ) through the Transitivity Update Module (TUM). UCUM is applied such that an adaptive and smooth transfer between two consecutive time windows (from current time-window to next time-window) is maintained. $\triangle T^{(i+1)}$ is the output of UCUM, and is calculated at the end of current time window $W^{(i)} . \triangle T^{(i+1)}$ is thus used in time window $W^{(i+1)}$ for the same tasks as in window $W^{(i)}$.

Forwarding Strategy Module (FSM) is applied at the custodian node as a forwarding decision making process when encountering any other node within the current time window based on the utility value (i.e., $\triangle T^{(i)}$ ).

It is important to note that CS, TUM, FSM, and message vector exchange are event-driven and performed during each contact, while UCUM is performed at the end of each time-window. The following subsections introduce each functional module in detail.

\subsection{Contact Statistics (CS)}

To compromise between the network state adaptability and computation complexity, each node continuously updates the network status over a fixed time window. The maintained network states are referred to as Contact Statistics (CS), which include nodal contact durations, channel conditions, and buffer occupancy state, and are fed into UCUM at the end of each time window. The CS collection process is described as follows.

Let two nodes $A$ and $B$ be in the transmission range of each other, and each broadcasts a pilot signal per $k$ time units in order to look for its neighbors within its transmission range. Let $T_{(A, B)}, T_{\text {free }}$, and $T_{\text {busy }}$ represent the total contact time, the amount of time the channel is free and the buffer is not full, and the amount of time the channel is busy or the buffer is full, respectively, at node $A$ or $B$ during time window $W^{(i)}$. Thus, the total duration of time in which node $A$ and $B$ can exchange information is calculated as:

$$
T_{\text {free }}=T_{(A, B)}-T_{\text {busy }}
$$

Note that the total contact time could be accumulated over multiple contacts between $A$ and $B$ during $W^{(i)}$.

\subsection{Utility-function Calculation and Update Module (UCUM)}

UCUM is applied at the end of each time window and is used to calculate the currently observed utility that will be further used in the next time window. The two inputs to UCUM in time window $W^{(i)}$ are: (i) the predicted inter-contact time $\left(\triangle T^{(i)}\right)$, which is calculated according to the previous time-window utility (i.e., $\triangle T^{(i)}$ ), as well as an update process via the transitivity property update (introduced in subsection 3.3), and (ii) the observed interencounter time obtained from the current $C S^{(i)}$ (denoted as $\left.\triangle T_{c s}^{(i)}\right)$.

\subsubsection{Calculation of Inter-encounter Time $\left(\triangle T^{(i)}\right)$}

An eligible contact of two nodes occurs if the duration of the contact can support a complete transfer of at least a single message between the two nodes. Thus, in the event that node $A$ encounters $B$ for a total time duration $T_{\text {free }}$ during time window $W^{(i)}$, the number of eligible contacts in the time window is determined by:

$$
n_{c}^{(i)}=\left\lfloor\frac{T_{\text {free }}}{T_{p}}\right\rfloor
$$

where $T_{p}$ is the least time duration required to transmit a single message. Let $\triangle T_{c s(A, B)}^{(i)}$ denotes the average inter-encounter time duration of node $A$ and $B$ in time $W^{(i)}$. Obviously, $\triangle T_{(A, B)}^{(i)}=\triangle T_{(B, A)}^{(i)}$. We have the following expression for $\triangle T_{c s(A, B)}^{(i)}$ :

$$
\triangle T_{c s(A, B)}^{(i)}=\frac{W^{(i)}}{n_{c}^{(i)}}
$$

$\triangle T^{(i)}{ }_{c s(A, B)}$ describes how often the two nodes encounter each other per unit of time (or, the encounter frequency) during time window $W^{(i)}$ considering the event the channel is busy or the buffer is full.

Thus, inter-encounter time of a node pair intrinsically relies rather on the duration and frequency of previous contacts of the two nodes than simply on the number of previous contacts or contact duration. Including the total duration of all the contacts (excluding the case when the channel is busy or the buffer is full) as the parameter is expected to better reflect the likelihood that nodes will meet with each other for effective message exchange.

With this, the proposed routing protocol does not presume any knowledge of future events, such as node velocity, node movement direction, instants of time with power on or off; instead, each node keeps network statistic histories with respect to the inter-encounter frequency of each node pair (or, how often the two nodes encounter each other and are able to perform an effective message exchange). 


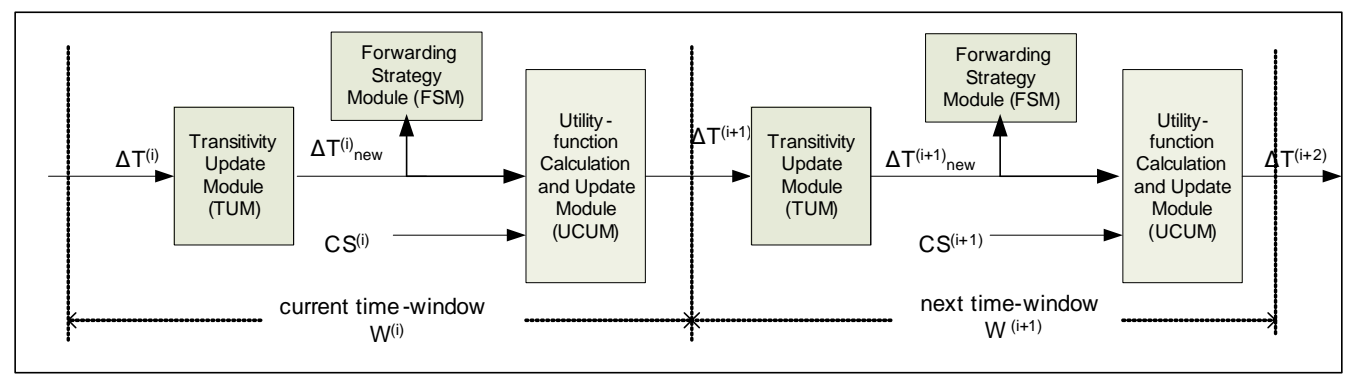

Fig. 1. The SAURP Architecture

\subsubsection{Time-window Transfer Update}

Another important function provided in UCUM is for the smooth transfer of the parameters between consecutive time windows. As discussed earlier, the connectivity between any two nodes is measured according to the amount of inter-encounter time during $W^{(i)}$, which is mainly based on the number of contacts (i.e., $n_{c}$ ) and the contact time (i.e., $T_{\text {free }}$ ). These contacts and contact durations may change dramatically from one time window to the other and address significant impacts on the protocol message forwarding decision. Hence, our scheme determines the next time window parameter using two parts: one is the current time window observed statistics (i.e., $\triangle T_{c s}^{(i)}$ ), and the other is from the previous time window parameters (i.e., $\left.\triangle T^{(i)}\right)$, in order to achieve a smooth transfer of parameter evolution. The following equation shows the derivation of $\triangle T^{(i+1)}$ in our scheme.

$$
\triangle T^{(i+1)}=\gamma \cdot \triangle T_{c s}^{(i)}+(1-\gamma) \triangle T^{(i)}
$$

The parameter $\gamma$ is given by

$$
\gamma=\frac{\left|\triangle T^{(i)}-\Delta T_{c s}^{(i)}\right|}{\max \left(\Delta T^{(i)}, \Delta T_{c s}^{(i)}\right)} \text {, where } \Delta T^{(i)}, \Delta T_{c s}^{(i)}>0
$$

If $\Delta T_{c s}^{(i)}>W$, which happens if if $n_{c}^{(i)}=0$, then $\triangle T^{(i+1)}=\frac{2 W}{n_{c}^{(i-1)}}$. This case represents a worst case scenario, i.e. unstable node behavior, or low quality of node mobility. Hence, the $\triangle T^{(i+1)}$ value should be low.

$\triangle T^{(i+1)}$ represents the routing metric (utility) value that is used as input to the next time window. This value is maintained as a vector of inter-encounter time that is specific to every other node, which is employed in the decision making process for message forwarding.

\subsection{The Transitivity Update Module (TUM)}

When two nodes are within transmission range of each other, they exchange utility vectors with respect to the message destination, based on which the custodian node decides whether or not each message should be forwarded to the encountered node. With a newly received utility vector, transitivity update [2] is initiated. We propose a novel adaptive transitivity update rule, which is different from the previously reported transitivity update rules [2]. [6]. The proposed transitivity update rule is characterized as follows: (1) it is adaptively modified according to a weighting factor $\alpha$, which is in turn based on the ratio of $\triangle T^{(i)}$ of the two encountered nodes regarding the destination rather than using a scaling constant. Note that the weighting factor $\alpha$ determines how large impact the transitivity should have on the utility function. (2) It can quantify the uncertainty regarding the position of the destination by only considering the nodes that can effectively enhance the accuracy of the utility function.

The transitivity property is based on the observation that if node $A$ frequently encounters node $B$ and $B$ frequently encounters node $D$, then $A$ has a good chance to forward messages to $D$ through $B$. Such a relation is implemented in the proposed SAURP using the following update strategy:

$$
\triangle T_{(A, D) \text { new }}^{(i)}=\alpha \triangle T_{(A, D)}^{(i)}+(1-\alpha)\left(\triangle T_{(A, B)}^{(i)}+\triangle T_{(B, D)}^{(i)}\right)
$$

where $\alpha$ is a weighting factor that must be less than 1 to be valid:

$$
\alpha=\frac{\triangle T_{(A, B)}^{(i)}+\triangle T_{(B, D)}^{(i)}}{\triangle T_{(A, D)}^{(i)}}, \triangle T_{(A, D)}^{(i)}>\triangle T_{(A, B)}^{(i)}+\triangle T_{(B, D)}^{(i)}
$$

$\alpha$ has a significant impact on the routing decision rule. From a theoretical perspective, when a node is encountered that has more information for a destination, this transitivity effect should successfully capture the amount of uncertainty to be resolved regarding the position of the destination.

To ensure that the transitivity effect can be successfully captured in the transitivity update process, an update should be initiated at node $A$ regarding $D$ only when $\triangle T_{(A, D)}^{(i)}>\triangle T_{(B, D)}^{(i)}$. Otherwise, the transitivity property for node $A$ is not useful since node $A$ itself is a better candidate for carrying the messages destined to node $D$ rather than forwarding them through $B$. This rule is applied after nodes finish exchange messages.

\subsection{The Forwarding Strategy Module (FSM)}

The decision of message forwarding in SAURP is mainly based on the utility function value of the encountered node regarding the destination, and the number of message copy tokens. If more than one message copy 
are currently carried, the weighted copy rule is applied; otherwise the forwarding rule is applied.

\subsubsection{Weighted Copy Rule}

The source of a message initially starts with $L$ copies. In the event that any node $A$ that has $n>1$ message copy tokens and encounters another node $B$ with no copies with $\triangle T_{(B, D)}^{(i)}<\triangle T_{(A, D)}^{(i)}$, node $A$ hands over some of the message copy tokens to node $B$ and keeps the rest for itself according to the following formula:

$$
N_{B}=\left\lfloor N_{A}\left(\frac{\triangle T_{(A, D)}^{(i)}}{\left.\triangle T_{(B, D)}^{(i)}+\triangle T_{(A, D)}^{(i)}\right)}\right)\right\rfloor
$$

where $N_{A}$ is the number of message tokens that node $A$ has, $\triangle T_{(B, D)}^{(i)}$ is the inter-encounter time between node $B$ and node $D$, and $\triangle T_{(A, D)}^{(i)}$ is the inter-encounter time between nodes $A$ and $D$. This formula guarantees that the largest number of message copies is spread to relay nodes that have better information about the destination node. After $L$ message copies have been disseminated to and carried by the encountered custodian nodes, each custodian node carrying the message performs message forwarding according to the forwarding rule as described in the next subsection. It may be noted here that the idea of weighted copy rule was firstly examined in [37] and our previous study [15], and has been proved to achieve improved delivery delay.

\subsubsection{The Forwarding Rule}

- If the destination node is one hop away from an encountered node, the custodian node hands over the message to the encountered node and completes the message delivery.

- If the inter-encounter time value of the encountered node relative to that of the destination node is less than that of the custodian node by a threshold value, $\triangle T_{t h}$, a custodian node hands over the message to the encountered node.

The complete mechanism of the forwarding strategy in SAURP is summarized as shown in Algorithm 1.

\section{Analytical Model of SaURP}

In this section a statistical analysis is conducted to evaluate the performance of SAURP. Without loss of generality, Community-Based Mobility Model [6] is employed in the analysis. The problem setup consists of an ad hoc network with a number of nodes moving independently on a 2-dimensional torus in a geographical region, and each node belongs to a predetermined community. Each node can transmit up to a distance $K \geq 0$ meters away, and each message forwarding (in one-hop) takes one time unit. Euclidean distance is used to measure the proximity between two nodes (or their positions) $A$ and $B$. A slotted collision avoidance MAC protocol with Clear-toSend (CTS) and Request-to-Send (RTS), is implemented for contention resolution. A message is acknowledged
Algorithm 1 The forwarding strategy of SAURP

On contact between node $A$ and $B$

Exchange summary vectors

for every message $M$ at buffer of custodian node $A$ do

if destination node $D$ in transmission range of $B$ then

$A$ forwards message copy to $B$

end if

else if $\triangle T_{(A, D)}^{(i)}>\triangle T_{(B, D)}^{(i)}$ do

if message tokens $>1$ then

end if

apply weighted copy rule

else if $\triangle T_{(A, D)}^{(i)}>\triangle T_{(B, D)}^{(i)}+\triangle T_{t h}$ then

$A$ forwards message to $B$ end else if

end else if

end for

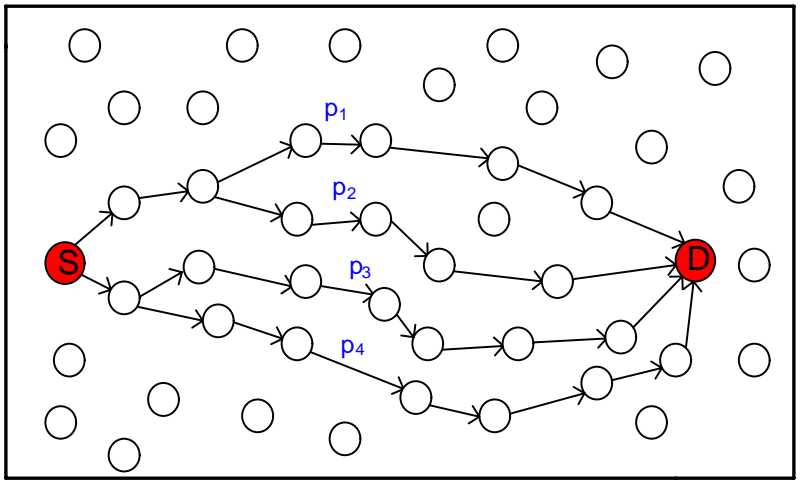

Fig. 2. Paths of message copies to destination

if it is received successfully at the encountered node by sending back a small acknowledgment packet to the sender.

The performance measures in the analysis include the average delivery probability and the message delivery delay. The analysis is based on the following assumptions.

- Nodes mobility is independent and heterogeneous, where nodes have frequent appearance in some locations.

- Each node in the network maintains at least one forwarding path to every other node. Fig. 2 illustrates the paths that a message copy may take to reach the destination.

- Each node belongs to a single community at a time (representing some hot spots such as classrooms, office buildings, coffee shops), and the residing time on a community is proportional to its physical size.

- The inter-contact time $\triangle T_{(A, B)}$ between nodes $A$ and $B$ follows an exponential distribution with probability distribution function (PDF), $P_{\triangle T_{(A, B)}}(t)=\beta_{(A, B)} \cdot e^{-\beta_{(A, B)} t}$, where $t$ is the time instance.

It has been shown that a number of popular user 
mobility models have such exponential tails (e.g., Random Walk, Random Waypoint, Random Direction, and Community-based Mobility [9], [26]). In practice, recent studies based on traces collected from real-life mobility examples argued that the inter-contact time and the contact durations of these traces demonstrate exponential tails after a specific cutoff point [18]. Based on the mobility model of the nodes, the distribution of the intercontact time can be predicted and calculated using time widow updates shown in (4). Thus, parameter $\beta_{A B}$ is calculated as $\beta_{A B}=\frac{1}{\triangle T_{(A, B)}}$.

\subsection{Delivery Probability}

In order to calculate the expected message delivery ratio, any path of message $m$ between $S$ and $D$ is a $k-h o p$ simple path, denoted as $l$, which is represented by a set of nodes and links denoted as $\left\{S, h_{1} h_{2} \ldots . h_{k-1}, D\right\}$, and $\left\{e_{1}, e_{2}, \ldots, e_{k}\right\}$, respectively. The cost on each edge, denoted as $\left\{\beta_{1}, \beta_{2}, . ., \beta_{k}\right\}$, is the inter-contact rate (or frequency) of each adjacent node pair along the path. According to the forwarding policy of SAURP, the values of inter-contact rate should satisfy $\left\{\beta_{1}<\beta_{2}<. .<\beta_{k}\right\}$. The path cost, $P R_{l}(t)$, is the probability that a message $m$ is successfully forwarded from $S$ to $D$ along path $l$ within time $t$, which represents a cumulative distribution function (CDF). The probability density function of a path $l$ with $k$-hop for one message copy can be calculated as convolution of $k$ probability distributions [27] which is calculated as:

$$
\operatorname{Pr}_{l}(t)=p_{1}(t) \otimes p_{2}(t) \otimes \ldots p_{k}(t)
$$

Theorem 1. Let the probability distribution function (PDF) for the message delivery along a one-hop path $i$ be denoted as $p_{i}(t)=\beta_{i} e^{-\beta_{i} t}$. Thus, the PDF for a $k-h o p$ simple path $l$ with an edge cost $\left\{\beta_{1}, \beta_{2}, \ldots, \beta_{k}\right\}$ can be expressed as

$$
\operatorname{Pr}_{l}(t)=\sum_{i=1}^{k_{l}} C_{i}^{\left(k_{l}\right)} p_{i}(t)
$$

where the coefficients are given as follows:

$$
C_{i}^{\left(k_{l}\right)}=\prod_{j=1, i \neq j}^{k_{l}} \frac{\beta_{j}}{\beta_{j}-\beta_{i}}
$$

The proof is provided in Appendix.

The probability of message delivery on forwarding path $l$ between any source $S$, and destination $D$, within expiration time $T$ is expressed as:

$$
\begin{aligned}
& F_{l}(T)=P R_{l}\left(T d_{l}<T\right)=\int_{0}^{T} \operatorname{Pr}_{l}(t) d t \\
& =\sum_{i=1}^{k_{l}} C_{i}^{k_{l}} \int_{0}^{T} P_{i}(t) d t \\
& \quad P R_{l}\left(T d_{l}<T\right)=\sum_{i=1}^{k_{l}} C_{i}^{\left(k_{l}\right)} \cdot\left(1-e^{-\beta_{i} T}\right)
\end{aligned}
$$

If there are $L-1$ copies (excluding the message at the source) of message $m$ traversing through $L-1$ independent paths in the network, the maximum probability of message delivery can be written as

$$
P R_{\max }\left(T_{d}<T\right)=\max \left\{P R_{S D}, P R_{1}, P R_{2}, . ., P R_{L-1}\right\}
$$

where $P R_{S D}$ and $P R_{l}$ are random variables representing the delivery probability in case of direct message delivery between $S$ and $D$, and through one of $L-1$ paths, respectively. The expected delivery probability of message $m$ with $L-1$ copies traversing on $L-1$ paths is calculated as:

$$
\begin{gathered}
P R\left(T_{d}<T\right)=1-P R_{S D}\left(T_{S D}>T\right) \prod_{l=1}^{L-1}\left(1-P R_{l}\left(T d_{l}<T\right)\right) \\
P R\left(T_{d}<T\right)=1-e^{-\beta_{S D} T} \prod_{l=1}^{L-1}\left(\sum_{i=1}^{k_{l}} C_{i}^{\left(k_{l}\right)}\left(e^{-\beta_{i} T}\right)\right)
\end{gathered}
$$

By assuming $X$ totally generated messages in the network, the average of the delivery probability in the network is calculated as

$$
P R=\frac{1}{X} \sum_{m=1}^{X} P R_{m}
$$

\subsection{Delivery Delay}

Theorem 2. The expected total time required to deliver a message from $S$ to $D$ along an individual path $l$ can be calculated as

$$
\begin{gathered}
E\left[D_{l}\right]=\int_{0}^{\infty} P R_{l}\left(T d_{l}>t\right)=\sum_{i=1}^{k_{l}} C_{i}^{\left(k_{l}\right)} \cdot \int_{0}^{\infty} e^{-\beta_{i} t} d t \\
E\left[D_{l}\right]=\sum_{i=1}^{k_{l}} C_{i}^{\left(k_{l}\right)} \cdot \frac{1}{\beta_{i}}
\end{gathered}
$$

Let message $m$ have $L-1$ copies (excluding the message at the source) traversing on $L-1$ independent paths. The minimum delivery delay can be written as:

$$
D_{S D}=\min \left\{T_{S D}, T d_{1}, T d_{2}, . . T d_{L-1}\right\}
$$

where $T_{S D}$ and $T d_{l}$ are a random variables representing the delivery delay through direct path between $S$ and $D$ and through one of $L-1$ paths, respectively. The expected delay of message $m, E\left[D_{S D}\right]$, can be calculated as

$$
\begin{gathered}
E\left[D_{S D}\right] \underset{0}{=} \quad \int_{0}^{\infty} P\left(T_{d}>\quad t\right)= \\
\int_{0}^{\infty} e^{-\beta_{S D} t} \prod_{l=1}^{L-1}\left(\sum_{i=1}^{k_{l}} C_{i}^{\left(k_{l}\right)} \cdot e^{-\beta_{i} t}\right) d t \\
=\frac{1}{\beta_{S D}} \int_{0}^{\infty} \beta_{S D} e^{-\beta_{S D} t} \prod_{l=1}^{L-1}\left(\sum_{i=1}^{k_{l}} C_{i}^{\left(k_{l}\right)} \cdot e^{-\beta_{i} t}\right) d t=
\end{gathered}
$$




$$
\frac{1}{\beta_{S D}} E\left\{\prod_{l=1}^{L-1}\left(\sum_{i=1}^{k_{l}} C_{i}^{\left(k_{l}\right)} \cdot e^{-\beta_{i} T_{S D}}\right)\right\}, T_{S D}<\infty
$$

The above relation gives an upper bound on the delivery delay since it is conditioned to $T_{S D}, T_{S D}<\infty$ and can be taken as point of reference.

The average delivery delay of message $m$ can be calculated intuitively as:

$$
E\left[E D_{(S, D)}\right]=\left[\frac{1}{L}\left(T_{S D}+\sum_{l=1}^{L-1} T d_{l}\right)\right] \cdot \frac{1}{P R\left(T_{d}<T\right)}
$$

$T_{S D}$ is included in (19) only if $T_{S D}<\infty$.

By assuming $X$ totally generated messages in the network, the average delivery delay can thus be calculated as

$$
D R=\frac{1}{X} \sum_{m=1}^{X} D_{m}
$$

\subsection{Validation of Analytical Model}

In order to evaluate the accuracy of the mathematical expressions in this analysis, SAURP is examined under two network status scenarios. In the first scenario, the network is operating under no congestion, i.e., all the nodes have infinite buffer space, and the bandwidth is much larger than the amount of data to be exchanged between any two encountered nodes. In the second scenario, the network is operating under limited resources, i.e., the forwarding opportunities can be lost due to high traffic, limited bandwidth, limited buffer space, or contention (i.e., more than one node within the transmission range are trying to access the wireless channel at the same time). For both scenarios, 50 nodes move according to community-based mobility model [6] in a 300x300 network area. The transmission range is set to 30 to enable moderate network connectivity with respect to the considered network size. The traffic load is varied from a low traffic load (i.e., 20 messages generated per node in 40,000 time units) to high traffic load (i.e., 80 messages generated per node in 40,000 time units). A source node randomly chosen a destination and generates messages to it during the simulation time. In this analysis the message copies are set to 5 (i.e., forming a maximum of 5 paths).

Examining SAURP under the two scenarios is very important; in case of no congestion, the best path that is taken by a message is mainly based on the interencounter time, while under congestion, the message will be buffered for longer period of time and enforced to take longer path to go around the congested area resulting in more dropping rate and longer delivery delay.

To enable accurate analysis, the simulation program is run for a period of time ( warm up period of 10,000

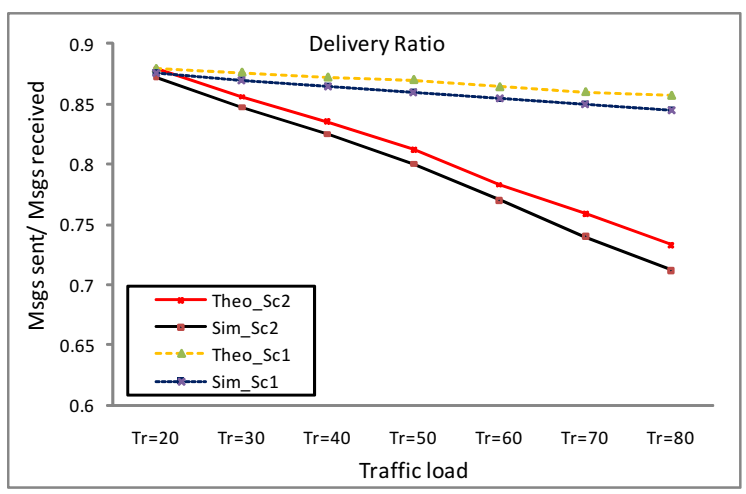

Fig. 3. The theoretical and simulation results of delivery ratio.

time units) such that each node can build and maintain the best forwarding paths with every other node in the network. These forwarding paths are mainly based on the congestion degree (traffic loads values) considered in the analysis. The forwarding path is cached by following the trajectories of the generated messages during the warm up stage between every source destination pair in the network. These messages are forwarded from node to node according to SAURP routing mechanism.

In this analysis, we simplified the calculation by limiting our study to only the best two of forwarding paths among all other paths and compare the simulation and theoretical results of delivery ratio and delivery delay. In most cases, a message takes the best forwarding path that based on the inter-encounters history if the network is not congested and the buffers operate under their capacity limit.

Fig. 3 and Fig. 4 compare the theoretical and simulation results in terms of delivery ratio and delivery delay of the considered scenarios.

As seen from the figures, when the network resources are enough to handle all the traffic loads (Scenario 1), there is no dramatic change in the obtained delivery ratio and delivery delay for all traffic loads. That is because messages follow the best forwarding paths that lead to best performance. The simulation and analytical plots for SAURP present close match and validates the generality of the analytical expressions. Additionally, it is evident that (16) and (20) are tight for all degrees of traffic loads. When the network resources are limited (i.e, scenario 2), the contention and the overhead of MAC layer increase, resulting in longer forwarding paths, higher drop rate, and longer delivery delay. The simulation and analytical plots are still providing close match with small diverge in case of high traffic loads.

Although the contention does affect the accuracy of our theoretical expressions, the error introduced for SAURP is not large $(20 \%)$, even for large traffic loads. Therefore, we believe the analytical expression is useful in assessing the performance in more realistic scenarios with contention. As an evident by these plots, the actual delay obtained by SAURP becomes increasingly worse 


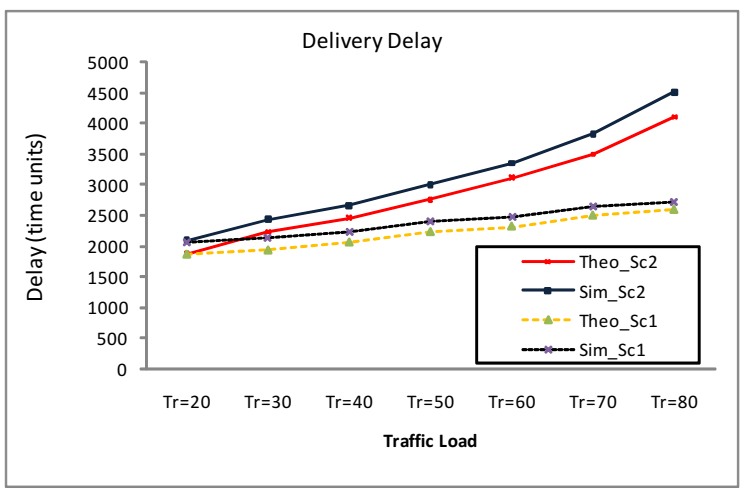

Fig. 4. The theoretical and simulation results of delivery delay.

than what the theory predicts. This demonstrates the need to add an appropriate contention model when it comes to modeling flooding-based schemes. A first effort in that direction can be found in [28].

\section{Performance Evaluation}

\subsection{Experimental Setup}

To evaluate the SAURP, a DTN simulator similar to that in [30] is implemented. The simulations are based on two mobility scenarios; a synthetic one based on community based mobility model (CBMM) [6], and a real-world encounter traces collected as part of the Infocome 2006 experiment, described in [42].

The problem setup consists of an ad hoc network with a number of nodes moving independently in a geographical region, and each node belongs to a predetermined community. Each node can transmit up to a distance $K \geq 0$ meters away, and each message transmission takes one time unit. A slotted collision avoidance MAC protocol with Clear-to-Send (CTS) and Request-to-Send (RTS), is implemented for contention resolution. A message is acknowledged if it is received successfully at the encountered node by sending back a small acknowledgment packet to the sender. The performance of SAURP is examined under different network scenarios and is compared with some previously reported schemes listed below.

- PROPHET [3]

- Spray and Focus (S\&F) [6]

- Most mobile first (MMF)[25]

- Delegation forwarding (DF) [14]

- Self-Adaptive utility-based routing protocol (SAURP)

- Self-Adaptive routing protocol (SARP) [15]

The performance comparison under the considered mobility scenarios is in terms of average delivery delay, delivery ratio, and the total number of transmissions performed for all delivered messages.

\subsection{CBMM Scenario}

\subsubsection{Evaluation Scenarios}

In the simulation, 110 nodes move according to the community-based mobility model [6] in a $600 \times 600$ meter network in a given geographical region. The simulation duration is 40,000 time. The message inter-arrival time is uniformly distributed in such a way that the traffic can be varied from low (10 messages per node in 40,000 time units) to high (70 messages per node in 40,000 time units). The message time to live (TTL) is set to 9,000 time units. Each source node selects a random destination node, begins generating messages to it during simulation time.

We analyze the performance implication of the following. First, the performance of the protocols is evaluated with respect to the impact of the number of message copies. Second, with respect to the low transmission range and varying buffer capacity under high traffic load. Third, with respect to the moderate-level of connectivity and varying traffic load. Fourth, the performance of the protocols is examined in terms of the bandwidth. Finally, the performance of the protocols is examined in terms of the level of connectivity changes.

\section{Impact due to Number of Message Copies}

We firstly look into impact of the number of message copies toward the performance of each protocol. The transmission range $K$ of each node is set to 30 meters, leading to a relatively sparse network. In order to reduce the effect of contention on any shared channel, the traffic load and buffer capacity is set to medium (i.e., 40 generated messages per node ) and high (i.e. 1,000 messages per node), respectively. The number of message copies is then increased from 1 to 20 in order to examine their impact on the effectiveness of each protocol. The proposed SAURP is compared with the S\&F and MMF schemes, since each scheme has a predefined $L$ to achieve the best data delivery. Note that the value of $L$ depends on the application requirements, the mobility model considered, and the design of the protocol.

Fig. 5 shows the results on message delivery delay, delivery ratio, and number of transmissions under different numbers of copies of each generated message. As can be seen, the $L$ value has a significant impact on the performance of each scheme. It is observed that best performance can be achieved under each scheme with a specific value $L$.

In the next scenarios, the number of message copies is fixed at 15 for the S\&F scheme, 10 for the SARP and SAURP, and 18 for the MMF. These $L$ values can serve as a useful rule of thumb for producing good performance.

\section{The Effect of Buffer Size}

In this scenario the performance of SAURP regarding different buffer sizes is examined under a low transmission range (i.e., $\mathrm{K}=30$ ) and a high traffic load (i.e., 50 

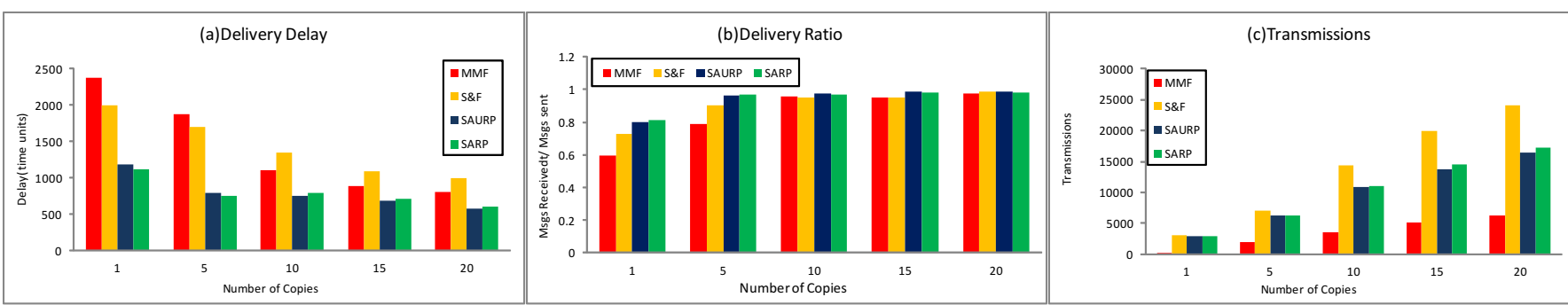

Fig. 5. Impact of the number of message copies

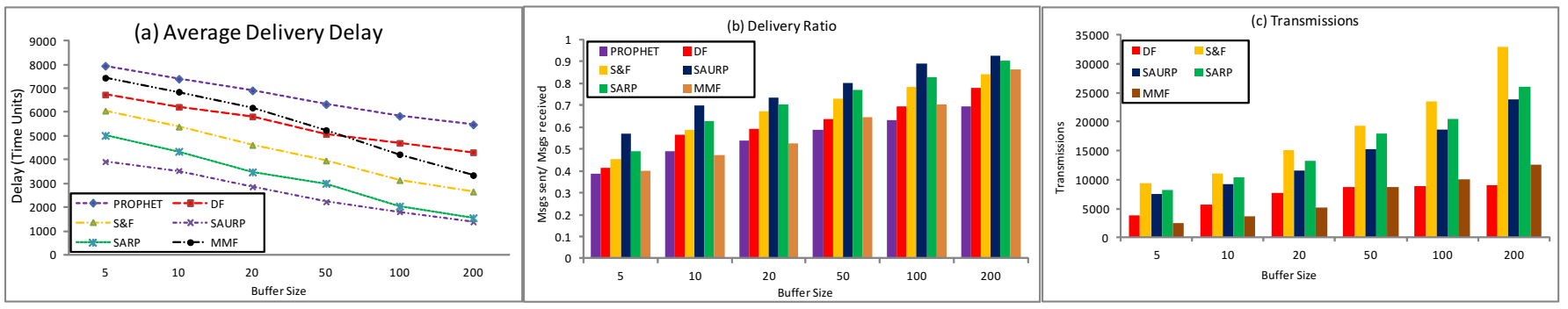

Fig. 6. The effect of buffer size
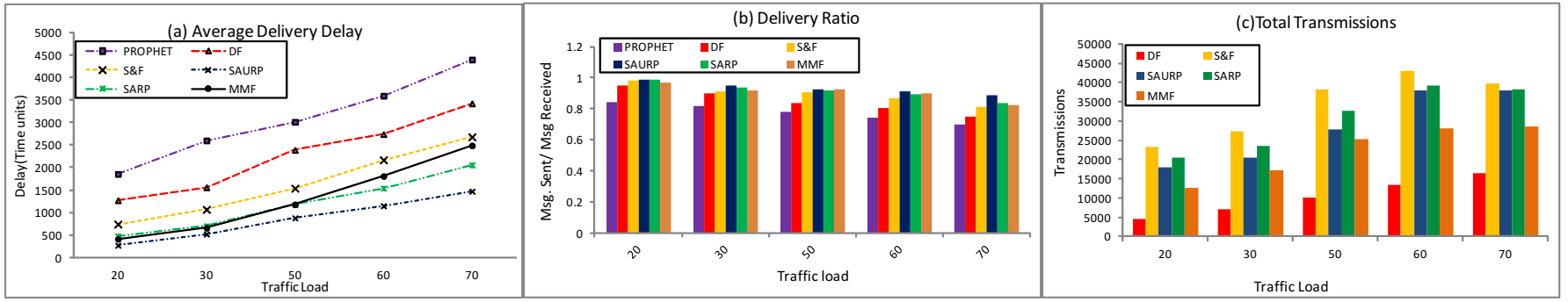

Fig. 7. The effect of traffic load under high buffer capacity
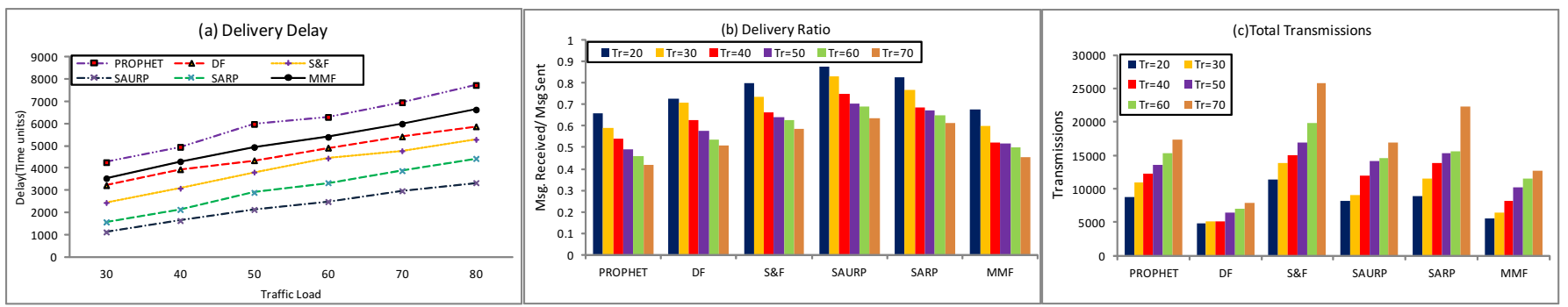

Fig. 8. The effect of traffic load under low buffer capacity

messages generated per node). Due to the high traffic volumes, we expect to see a significant impact upon the message forwarding decisions due to the degradation of utility function values caused by buffer overflow. Note that when the buffer of the encountered node is full, some messages cannot be delivered even though the encountered node metric is better than the custodian node. This situation results in extra queuing delay, especially in the case that flooding-based schemes are in place. Fig. 6 shows the experiment results where the buffer space was varied from 5 (very limited capacity) to 200 (relatively high capacity) messages to reflect the performance of the protocols under the considered traffic load. As shown in Fig. 6, when the buffer size is small (50 messages or less) the performance of the protocols is very sensitive to the change of buffer capacity.

It is observed that the SAURP scheme produced the best performance in all scenarios, since it takes the situation that a node may have a full buffer into consideration by degrading the corresponding utility metric, it produced the best performance. In specific, SAURP yielded a shorter delivery delay than that of PROPHET by $230 \%$, S\&F by $50 \%$, and SARP by $22 \%$. SAURP can achieve a higher delivery ratio than DF by $73 \%$, PROPHET by $79 \%$, S\&F by $66 \%$, and SARP by $17 \%$. Although SUARP produced more transmissions than MMF and DF, it yielded a smaller delivery delay than that of MMF by $82 \%$, and DF by $66 \%$. As the buffer 
size increased, the performance of all protocols improved especially for MMF and SARP. When the buffer size is larger than the traffic demand, the SARP scheme has yielded a competitive performance due to the relaxation of buffer capacity limitation. SAURP still yielded the best performance with a smaller number of transmissions than $S \& F$ by $33 \%$.

\section{The Effect of Traffic Load}

The main goal of this scenario is to observe the performance impact and how SAURP reacts under different degrees of wireless channel contention. The network connectivity is kept high (i.e., the transmission range is set to as high as 70 meters) under different traffic loads, while channel bandwidth is set relatively quite small (i.e., one message transfer per unit of time) in order to create congested environment. We have two scenarios for nodal buffer capacity: 1) unlimited capacity; and 2) low capacity (15 messages). Fig. 7 shows the performance of all the routing algorithms in terms of the average delivery delay, delivery ratio, and total number of transmissions.

It is observed that PROPHET produced the largest delivery delay and requires a higher number of transmissions compared to all the other schemes, thus it is not included in figure 7(c). PROPHET produced an order of magnitude more transmissions than that by SAURP.

As shown in Fig. 7(a), 7(b), and 7(c), when the traffic load is increased, the available bandwidth is decreased accordingly, which causes performance reduction. When the traffic load is moderate (i.e., less that 50 messages), it is clear that the delivery delay is short in all the schemes, while SAURP outperforms all other protocols and MMF is the second best. This is because in MMF, the effect of buffer size is relaxed, which makes nodes buffer an unlimited number of messages while roaming among communities. SAURP can produce delay shorter than that of PROPHET, MMF, DF, S\&F, and SARP by $350 \%$, $52 \%, 400 \%, 250 \%$, and $57 \%$, respectively. Regarding the delivery ratio, SAURP, MMF, S\&F, and SARP can achieve excellent performance of $98 \%$, while the PROPHET routing degrades below $60 \%$ for high traffic loads. DF can achieve delivery ratio above $92 \%$.

As expected, the performance of all the schemes degrades as wireless channel contention is getting higher, especially when the traffic load exceeds 50 messages per node during the simulation period. We observed that SAURP can achieve significantly better performance compared to all the other schemes, due to the consideration of busy links in its message forwarding mechanism, where the corresponding routing-metric is reduced accordingly. This results in the ability of rerouting the contended messages through the areas of low congestion. However, such a rerouting mechanism makes messages take possibly long routes and results in more transmissions than that of MMF and DF. In summary, the delivery delay obtained by the SAURP in this scenario is shorter than that of PROPHET by $330 \%$, MMF by $66 \%$, S\&F by $88 \%$, DF by $233 \%$, and SARP by $30 \%$ respectively. Regarding delivery ratio, SAURP can achieve as high as $93 \%$, compared with $90 \%$ by SARP, $87 \%$ by MMF, $77 \%$ by $\mathrm{DF}$, and $88 \%$ by S\&F. Even though DF produced the lowest number of transmissions, it is at the expense of the worst delivery delay.

As the buffer capacity is low (e.g., 15 messages) and the traffic load is high, the available bandwidth decreases and the buffer occupancy increases accordingly, which makes the performance of all protocols degraded, especially for the PROPHET and MMF. It is observed that PROPHET produced the largest delivery delay. It is notable that SAURP outperforms all the multiple-copy routing protocols in terms of delivery delay and delivery ratio under all possible traffic loads. When the traffic load is high, SAURP yielded shorter delivery delay than that of SARP by $28 \%$, MMF by $53 \%$, SF by $41 \%$, DF by $47 \%$, and PROPHET by $233 \%$. Although SAURP requires more transmissions compared to the MMF and $\mathrm{DF}$, the number is still smaller than that produced by S\&F. SAURP can achieve delivery ratio above $76 \%$ for high traffic loads, while the SARP, PROPHET, DF, $\mathrm{S} \& \mathrm{~F}$, and MMF degrades by $66 \%, 47 \%, 51 \%, 62 \%$, and $55 \%$, respectively. Fig. 8(a), 8(b), and 8(c) shows the performance of all techniques under this scenario.

\section{The Effect of Channel Bandwidth and Traffic Load}

To examine the effect of channel bandwidth, the network connectivity is set to moderate (under moderate transmission range by setting $K=50$ ), and the link capacity is set five times higher than that used in the previous scenarios in order to avoid bottlenecks in the traffic loads. Fig. 9 shows the performance of all the routing protocols in terms of the average delivery delay, delivery ratio, and total number of transmissions.

As the link bandwidth increased, it can be seen from Fig. 9 that the performance of all routing schemes has improved with respect to delivery delay and delivery ratio, because the buffer capacity is unlimited and the contention on the bandwidth is relaxed. SARP achieved the best performance, while SAURP achieves the second best compared to the other schemes. It outperforms MMF scheme, since MMF is coupled by the number of message copies. Compared to PROPHET, DF, and S\&F, SAURP has a shorter delay by $450 \%, 390 \%$, and $83 \%$, respectively. Meanwhile, SAURP needed much less transmissions compared to that by S\&F. Even though DF produced the lowest number of transmissions, it has the worst performance in terms of delivery delay and delivery ratio. All protocols achieved a delivery ratio above $90 \%$. Compared to other protocols, SAURP maintains the second highest delivery ratio after SARP: above $96 \%$.

The above results show that channel bandwidth has significant impact on the performance of the protocols. If the available bandwidth is much higher than the total traffic load, flooding based schemes [11] can yield delivery delay as SAURP at the expense of taking far 

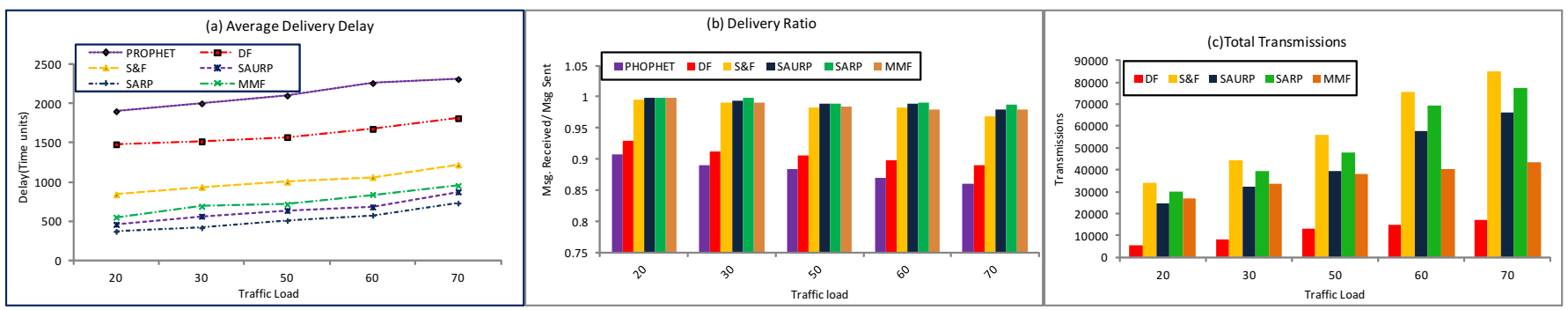

Fig. 9. The effect of traffic load under high link bandwidth

more transmissions. On the other hand, if the channel bandwidth is limited, SAURP and the spraying schemes outperform the flooding-based schemes because of the contention caused by limited bandwidth.

\section{The Effect of Connectivity}

This scenario studies the performance impact due to network topology connectivity. In the scenario, the level of connectivity is increased from very sparse to highly connected by varying the value of $K$ while observing the resultant impact on the performance. We are particularly interested to investigate the SAURP mechanism in response to heavy traffic loads which result in high contention on the wireless channel. The buffer capacity is kept low (15 messages), and the traffic load is considerably high (60 messages per node). Fig. 10 shows the average delay, delivery ratio, and the number of transmissions as a function of transmission range.

SAURP outperforms all the schemes in terms of delivery delay while taking noticeably fewer transmissions than that by $S \& F$ and and SARP schemes under all connectivity considered in the simulation. When the network is sparsely connected, SAURP can achieve shorter delivery delay than all other schemes, that is because the performance of other schemes is affected by the uncertainty of buffer occupancy status. On the other hand, when the network is moderate-connected, SARP can achieve commutative-level of delivery delay compared to SAURP with more transmissions. As the network becomes almost connected and the traffic load is high, the uncertainty of both buffer occupancy status and the availability of bandwidth affect the performance of the other techniques. As a result, SAURP outperforms all other schemes in terms of delivery delay and delivery ratio.

\subsection{Real Trace Scenario}

In order to evaluate SAURP in realistic environment, the performance of the scheme is examined using real encounter traces. These data sets comprise of contact traces between short-range Bluetooth enabled devices carried by individuals in Infocome 2006 conference environment. More details about the devices and the data sets, including synchronization issues can be found in [42]. In order to observe the performance impact and how SAURP reacts under congested environment, we set the bandwidth, buffer capacity, and the distribution of the contact time such that congested environment is formed. The channel bandwidth is set relatively quite small (i.e., one message transfer per unit of time), and the buffer size is set to 10, under different levels of traffic demand.

Fig. 11 shows the performance of all the routing algorithms in terms of the average delivery delay, delivery ratio, and total number of transmissions.

As the buffer capacity is low and the traffic load is high, the available bandwidth decreases and the buffer occupancy increases accordingly, which makes the performance of all protocols degraded, especially for the PROPHET and MMF. It is observed that PROPHET produced the largest delivery delay. It is subject to at least 2.1 times of longer delivery delay than that by SARP. It is notable that SAURP outperforms all the multiplecopy routing protocols in terms of delivery delay and delivery ratio under all possible traffic loads. When the traffic load is high, SAURP yielded shorter delivery delay than that of MMF by $52 \%$, SF by $30 \%$, and DF by $40 \%$. Although SAURP requires more transmissions compared to the MMF and DF, the number is still smaller than that produced by S\&F. SAURP can achieve delivery ratio above $76 \%$ for high traffic loads, while the SARP, POPHET, DF, S\&F, and MMF degrades by $67 \%$, 38\%, $53 \%, 60 \%$, and $50 \%$, respectively.

\section{Conclusion}

The paper introduced a novel multi-copy routing scheme, called SAURP, for intermittently connected mobile networks that are possibly formed by densely distributed and hand-held devices such as smart phones and personal digital assistants. SAURP aims to explore the possibility of taking mobile nodes as message carriers in order for end-to-end delivery of the messages. The best carrier for a message is determined by the prediction result using a novel contact model, where the network status, including wireless link condition and nodal buffer availability, are jointly considered. We provided an analytical model for SAURP, whose correctness was further verified via simulation. We further compared SAURP with a number of counterparts via extensive simulations. It was shown that SAURP can achieve shorter delivery delays than all the existing spraying and flooding based schemes when the network experiences considerable 


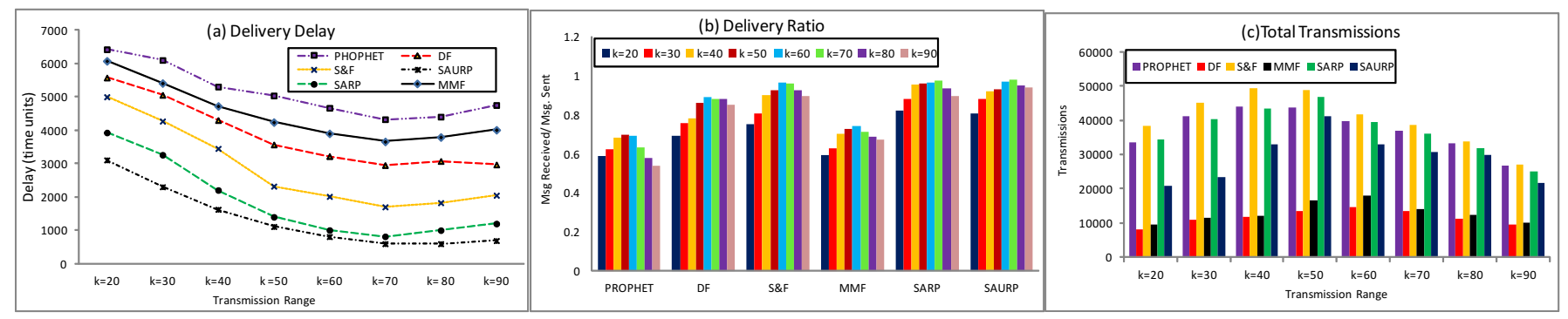

Fig. 10. The effect of connectivity

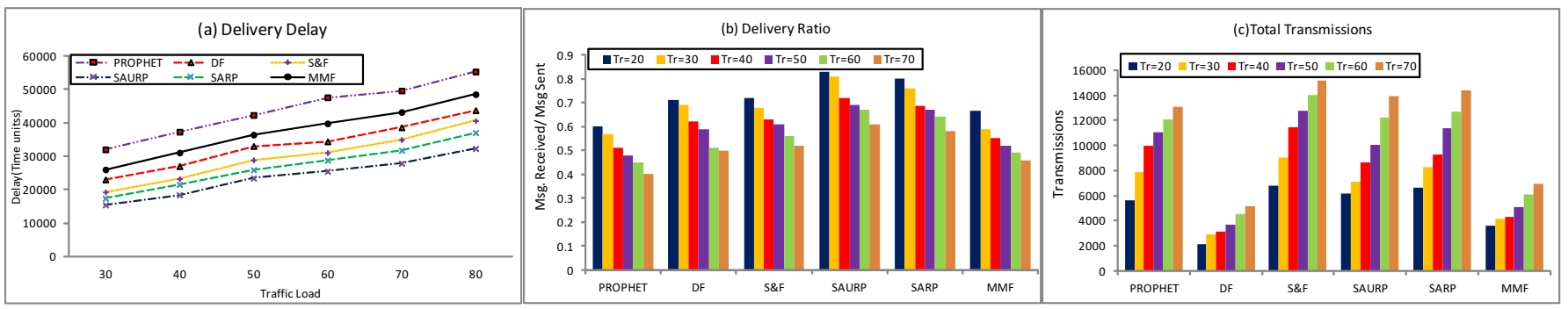

Fig. 11. The effect of traffic load under trace based scenario

contention on wireless links and/or buffer space. The study provides a significance that when nodal contact does not solely serve as the major performance factor, the DTN routing performance can be significantly improved by further considering other resource limitations in the utility function and message weighting/forwarding process.

\section{APPENDIX}

Proof of (10):

First we compute the convolution needed in the proof.

$$
\begin{aligned}
& e^{-a x} \otimes e^{-b x}=\int_{0}^{x} e^{-a(x-u)} e^{-b u} d u=e^{-a x} \frac{e^{(a-b) x}-1}{a-b} \\
& =\frac{e^{-b x}-e^{-a x}}{a-b}
\end{aligned}
$$

for two hop $(k=2)$

$$
\begin{aligned}
& P_{1+2}=P_{1} \otimes P_{2}=\beta_{1} \beta_{2} \int_{0}^{x} e^{-\left(\beta_{1}-\beta_{2}\right) t} e^{-\beta_{2} x} d t \\
& =\frac{\beta_{1} \beta 2}{\beta_{2}-\beta_{1}}\left(e^{-\left(\beta_{1} x-\beta_{2} x\right)}\right)=\beta_{1} \beta 2\left[\frac{e^{-\beta_{1} x}}{\beta_{2}-\beta_{1}}+\frac{e^{-\beta_{2} x}}{\beta_{1}-\beta_{2}}\right] \\
& =-C_{1}^{(2)} P_{1}(x)-C_{2}^{(2)} P_{2}(x)
\end{aligned}
$$

For $k \geq 3$, by inductive we can get

$$
\begin{aligned}
& P_{k-1}=\sum_{i=1}^{k_{l}-1} C_{i}^{k_{l}-1} \cdot P_{i}(x) \\
& P_{k}=P_{1+2+\ldots k-1} \otimes P_{k}= \\
& {\left[\prod_{i=1}^{k-1} \beta_{i}\right] \sum_{j=1}^{k_{l}-1} \frac{e^{\beta_{j} x}}{\prod_{i=1}^{k-1}\left(\beta_{k}-\beta_{j}\right)} \otimes P_{k}} \\
& =\sum_{i=1}^{k_{l}-1} C_{i}^{k_{l}-1}\left(\frac{\beta_{k}}{\beta_{k}-\beta_{i}} P_{i}(x)+\frac{\beta_{i}}{\beta_{i}-\beta_{k}} P_{k}(x)\right)
\end{aligned}
$$

If we consider $C_{i}^{k_{l}}=C_{i}^{k_{l}-1} \cdot \frac{\beta_{i}}{\beta_{i}-\beta_{k}}$, we get
$P_{k}=\sum_{i=1}^{k_{l}-1} C_{i}^{k_{l}} \cdot P_{i}(x)+\sum_{i=1}^{k_{l}-1} C_{i}^{k_{l}-1} \cdot \frac{\beta_{i}}{\beta_{i}-\beta_{k}} P_{k}(x)$

For the second term, we have

$\sum_{i=1}^{k_{l}-1} C_{i}^{k_{l}-1} \frac{\beta_{i}}{\beta_{i}-\beta_{k}}=\sum_{i=1}^{k_{l}-1} \frac{\beta_{i}}{\beta_{i}-\beta_{k}}$

$\cdot\left(\prod_{j=1, j \neq i}^{k-1} \frac{\beta_{j}}{\beta_{j}-\beta_{i}}\right)$

$=\prod_{j=1}^{k-1} \beta_{j} \cdot \sum_{i=1}^{k_{l}-1} \prod_{j=1, j \neq i}^{k-1} \frac{1}{\beta_{j}-\beta_{i}}$

$=\prod_{j=1}^{k-1} \frac{\beta_{j}}{\beta_{j}-\beta_{k}}=C_{k}^{k_{l}}$

Therefore, we have

$f_{k}(x)=P_{k}=\sum_{i=1}^{k_{l}-1} C_{i}^{k_{l}} P_{i}(x)+C_{k}^{k_{l}} P_{k}(x)$

$=\sum_{i=1}^{k_{l}} C_{i}^{k_{l}} P_{i}(x)$

CDF:

$F_{k}(x)=P\left(T_{d}<T\right)=\int_{0}^{T} f_{k}(x) d x=$

$\sum_{i=1}^{k_{l}} C_{i}^{k_{l}} \int_{0}^{T} P_{i}(x) d x=\sum_{i=1}^{k_{l}} C_{i}^{k_{l}} \cdot\left(1-e^{-\beta_{i} T}\right)$

CCDF:

$=P\left(T_{d}>T\right)=\int_{T}^{\infty} f_{k}(x) d x=\sum_{i=1}^{k_{l}} C_{i}^{k_{l}} \cdot e^{-\beta_{i} T}$

\section{REFERENCES}

[1] Disruption Tolerant Networking. http://www.dtnrg.org.

[2] A. Lindgren, A. Doria, and O. Schelen. Probabilistic routing in intermittently connected networks. SIGMOBILE Mobile Comput. Commun. Rev., 7(3):19-20, 2003.

[3] A. Vahdat and D. Becker, "Epidemic Routing for Partially Connected Ad Hoc Networks ," Duke University, Tech. Rep. CS-200006, July 2000. 
[4] Y.-C. Tseng, S.-Y. Ni, Y.-S. Chen, and J.-P. Sheu. "The broadcast storm problem in a mobile ad hoc network". Wireless Networks, $8(2 / 3), 2002$

[5] T. Spyropoulos, K. Psounis, and C. S. Raghavendra. "Efficient Routing in Intermittently Connected Mobile Networks: The Single-copy Case," IEEE Trans. Networking Vol. 16, Issue. 1, Feb. 2008

[6] _ "Efficient routing in intermittently connected mobile networks: The Multi-copy case," IEEE Trans. Networking vol. 16, Issue 1, Feb. 2008.

[7] Z. Li and Haiying Shen. Utility-based Distributed Routing in Intermittently Connected Networks. 37th International Conference on Parallel Processing. (ICPP '08).September 8-12, 2008, Portland, Oregon, USA.

[8] H. Dubois-Ferriere, M. Grossglauser, and M. Vetterli. Age matters: efficient route discovery in mobile ad hoc networks using encounter ages. In Proceedings of ACM/IEEE MOBIHOC, pages 257-266, 2003.

[9] T. Spyropoulos, K. Psounis, and C. S. Raghavendra. "Performance analysis of mobility-assisted routing," in Proc. ACM/IEEE MOBIHOC, 2006.

[10] Z. Zhang. Routing in Intermittently Connected Mobile Ad Hoc Net-works and Delay Tolerant Networks: Overview and Challenges. IEEE Communications Surveys and Tutorials, 8(1).

[11] M. Musolesi, S. Hailes, and C. Mascolo.Adaptive routing for intermittently connected mobile ad hoc networks. In Proceedings of IEEE WoWMoM, June 2005, pp. 183-189.

[12] T. Spyropoulos, K. Psounis, and C. S. Raghavendra. "Utility-based Message Replication for Intermittently Connected Heterogeneous Wireless Networks", in Proceedings of IEEE WoWMoM workshop on Autonomic and Opportunistic Communications (AOC), (INRIA Technical Report RR-6129), June 2007.

[13] J. Broch, D. A. Maltz, D. B. Johnson, Y.-C. Hu, and J. Jetcheva. A performance comparison of multi-hop wireless ad hoc network routing protocols. Proc. ACM MobiCom. Oct. 1998

[14] V. Erramilli, M. Crovella, A. Chaintreau , C. Diot. Delegation forwarding. Proceedings of the 9 th ACM international symposium on Mobile ad hoc networking and computing, May 26-30, 2008, Hong Kong, Hong Kong, China.

[15] Ahmed Elwhishi and Pin-Han Ho. 2009. SARP - a novel multi-copy routing protocol for intermittently connected mobile networks. In Proceedings of the 28th IEEE conference on Global telecommunications (GLOBECOM'09), Mehmet Ulema (Ed.). IEEE Press, Piscataway, NJ, USA, 4482-4488.

[16] A. Elwhishi Pin-Han Ho, K. Naik, and B. Shihadda. ARBR: Adaptive Reinforcement-Based Routing for DTN. WiMob 2010. Oct. 11-14: 376-385.

[17] Musolesi, M., and Mascolo, C. 2009. CAR: Context-aware Adaptive Routing for Delay Tolerant Mobile Networks. IEEE Transactions on Mobile Computing. 8, 2 (February 2009), 246-260.

[18] M. V. Thomas Karagiannis, Jean-Yves Le Boudec, "Power law and exponential decay of inter contact times between mobile devices," in Proc. of ACM/IEEE MobiCom, 2007.

[19] Evan P. C. Jones,Lily Li, and Paul A. S. Ward. Practical Routing in Delay-Tolerant Networks.In SIGCOMM'05 Workshops, August 22-26, 2005, Philadelphia, PA, USA.

[20] T. Spyropoulos, K. Psounis, and C. Raghavendra. 2005. Spray and wait: an efficient routing scheme for intermittently connected mobile networks. In Proceedings of the 2005 ACM SIGCOMM workshop on Delay-tolerant networking (WDTN '05)

[21] Q. Yuan, I. Cardei, and J. Wu. 2009. Predict and relay: an efficient routing in disruption-tolerant networks. In Proceedings of the tenth ACM international symposium on Mobile ad hoc networking and computing (MobiHoc '09).

[22] A. Balasubramanian, B. Levine , A. Venkataramani, DTN routing as a resource allocation problem, Proceedings of the 2007 conference on Applications, technologies, architectures, and protocols for computer communications, August 27-31, 2007, Kyoto, Japan.

[23] Shen Ling, Wu Wei, "Feedback Adaptive Routing Algorithm for DTN," cmc, vol. 2, pp.267-271, 2009 WRI International Conference on Communications and Mobile Computing, 2009.

[24] V. Erramilli, M. Crovella. Forwarding in opportunistic Networks with Resource constraints. CHANTS'08, September 15, 2008, San Francisco, California, USA.

[25] T. Spyropoulos, T. Turletti, and K. Obraczka, "Routing in delaytolerant networks comprising heterogeneous node populations,"
IEEE Transactions on Mobile Computing, vol. 8, no. 8, pp. 1132-1147, August 2009.

[26] R. Groenevelt, P. Nain, and G. Koole. The message delay in mobile ad hoc networks. In Performance, October 2005.

[27] Akkouchi M. On the convolution of exponential distributions. J. Chungcheong Math. Soc, vol. 21 no.4. pp.501-510, 2008.

[28] A. Jindal and K. Psounis. Contention-aware performance analysis of mobility-assisted routing. IEEE Transactions on Mobile Computing, February 2009.

[29] Samuel C Nelson, Mehedi Bakht, Robin Kravets, and Albert F. Harris, III. 2009. Encounter: based routing in DTNs. SIGMOBILE Mob. Comput. Commun. Rev. 13, 1 (June 2009), 56-59.

[30] "DTN java simulator," http://people.ee.ethz.ch/ spyropot/dtnsim.html.

[31] Daly, E.M.; Haahr, M.; , "Social Network Analysis for Information Flow in Disconnected Delay-Tolerant MANETs," Mobile Computing, IEEE Transactions on , vol.8, no.5, pp.606-621, May 2009 doi: 10.1109/TMC.2008.161

[32] E.Daly and M.Haahr. Social Network Analysis for Routing in Disconnected Delay-Tolerant MANETs. MobiHoc'07, ACM 9781-59593-684-4/07/0009, September 2007

[33] Jill Freyne, Shlomo Berkovsky, Elizabeth M. Daly, and Werner Geyer. 2010. Social networking feeds: recommending items of interest. In Proceedings of the fourth ACM conference on Recommender systems (RecSys '10). ACM, New York, NY, USA, 277-280.

[34] P. Hui, J. Crowcroft, and E. Yoneki, "Bubble rap: Social-based forwarding in delay tolerant networks," in MobiHoc '08: Proceedings of the 9th ACM international symposium on Mobile ad hoc networking \& computing, May 2008.

[35] T. Abdelkader, K. Naik, A. Nayak, and N. Goel, "A SociallyBased Routing Protocol for Delay Tolerant Networks", in Proc. GLOBECOM, 2010, pp.1-5.

[36] ] K. Tan, Q. Zhang, and W. Zhu, "Shortest path routing in partially connected ad hoc networks," in Globecom, 2003.

[37] Yong Liao, el at, cooperative robust forwarding scheme in DTNs using erasure coding, MILCOM 2007

[38] MaxProp: J. Burgess, B. Gallagher, D. Jensen, and B. N. Levine, "MaxProp: Routing for vehicle-based disruption-tolerant networks," in Proc. of INFOCOM, 2006.

[39] K. Lee, Y. Yi, J. Jeong, H. Won, I. Rhee, and S. Chong, "MaxContribution: On optimal resource allocation in delay tolerant networks."in Proc. of INFOCOM, 2010.

[40] MOPS: F. Li and J. Wu, "Mops: Providing content-based service in disruptiontolerant networks," in Proc. of ICDCS, 2009, pp. 526-533.SimBet: E. M. Daly and M. Haahr, "Social network analysis for routing in disconnected delay-tolerant manets," in Proc. of MobiHoc, 2007.

[41] B. Chiara, C. Marco, J. Jacopo, and P. Andrea, "Hibop: A history based routing protocol for opportunistic networks," in Proc. of WoWMoM2007,2007, pp. 1-12.

[42] CHAINTREAU, A., HUI, P., CROWCROFT, J., DIOT, C., GASS, R., AND SCOTT, J. Impact of Human Mobility on Opportunistic Forwarding Algorithms. IEEE Trans. on Mobile Computing 6, 6 (2007), 606-620.

[43] A. Krifa, C. Barakat, and T. Spyropoulos. Optimal buffer management policies for delay tolerant networks. In Proc. of IEEE SECON, 2008. 